\title{
Alterstice
}

Revue internationale de la recherche interculturelle

International Journal of Intercultural Research

Revista International de la Investigacion Intercultural

\section{L'école à l'épreuve de la diversité ethnoculturelle de son personnel : regards compréhensifs croisés}

\section{Joëlle Morrissette et Geneviève Audet}

Volume 8, numéro 2, 2018

L'école à l'épreuve de la diversité ethnoculturelle de son personnel : regards compréhensifs croisés

URI : https://id.erudit.org/iderudit/1066948ar

DOI : https://doi.org/10.7202/1066948ar

Aller au sommaire du numéro

Éditeur(s)

Alterstice

ISSN

1923-919X (numérique)

Découvrir la revue

Citer ce document

Morrissette, J. \& Audet, G. (2018). L'école à l'épreuve de la diversité ethnoculturelle de son personnel : regards compréhensifs croisés. Alterstice, 8(2), 5-12. https://doi.org/10.7202/1066948ar

\section{Résumé de l'article}

Ce numéro d'Alterstice s'inscrit dans le contexte actuel, où les rencontres interculturelles sont multipliées. La diversification des société fait naître différentes problématiques, notamment celles liées à l'intégration sociale et professionnelle des immigrants et de leurs familles, en particulier dans les grands centres urbains, où ils ont davantage tendance à s'établir. Mais c'est à l'école, dans les établissements scolaires et leurs environnements, lieux privilégiés de rencontres, de maillage et de métissage, que cet enjeu prend forme, dans des dynamiques de socialisation aux codes du vivre ensemble. Nous interrogeons ici les expériences et relations interculturelles au sein de la communauté éducative, ce qui implique de porter attention à une multiplicité d'acteurs : enseignants, élèves, professionnels (orthopédagogues, éducateurs spécialisés, etc.), équipe de direction, employés de soutien (secrétaire, concierge, etc.), familles, organismes communautaires, etc. Divers éclairages théoriques et empiriques sont proposés pour appréhender les processus d'ajustements continus et réciproques dans la négociation de cette diversité à l'école, au travers des interactions quotidiennes. Sont regroupés d'abord les textes qui rapportent spécifiquement la perspective des enseignants formés à l'étranger à propos de leurs expériences d'intégration dans le nouveau milieu professionnel, au Québec et en Ontario. Sont ensuite proposés des textes qui croisent les regards de différents acteurs sur la diversité ethnoculturelle au sein de l'école et les défis qu'elle soulève, mais aussi les ressources qu'elle constitue pour les relations au sein de l'écologie professionnelle.
Ce document est protégé par la loi sur le droit d'auteur. L'utilisation des services d’Érudit (y compris la reproduction) est assujettie à sa politique d'utilisation que vous pouvez consulter en ligne.

https://apropos.erudit.org/fr/usagers/politique-dutilisation/ 


\section{7}

INTRODUCTION THÉMATIQUE

\section{L'école à l'épreuve de la diversité ethnoculturelle de son personnel : regards compréhensifs croisés}

Joëlle Morrissette ${ }^{1}$ et Geneviève Audet ${ }^{2}$

\section{Résumé}

Ce numéro d'Alterstice s'inscrit dans le contexte actuel, où les rencontres interculturelles sont multipliées. La diversification des société fait naître différentes problématiques, notamment celles liées à l'intégration sociale et professionnelle des immigrants et de leurs familles, en particulier dans les grands centres urbains, où ils ont davantage tendance à s'établir. Mais c'est à l'école, dans les établissements scolaires et leurs environnements, lieux privilégiés de rencontres, de maillage et de métissage, que cet enjeu prend forme, dans des dynamiques de socialisation aux codes du vivre ensemble. Nous interrogeons ici les expériences et relations interculturelles au sein de la communauté éducative, ce qui implique de porter attention à une multiplicité d'acteurs : enseignants, élèves, professionnels (orthopédagogues, éducateurs spécialisés, etc.), équipe de direction, employés de soutien (secrétaire, concierge, etc.), familles, organismes communautaires, etc. Divers éclairages théoriques et empiriques sont proposés pour appréhender les processus d'ajustements continus et réciproques dans la négociation de cette diversité à l'école, au travers des interactions quotidiennes. Sont regroupés d'abord les textes qui rapportent spécifiquement la perspective des enseignants formés à l'étranger à propos de leurs expériences d'intégration dans le nouveau milieu professionnel, au Québec et en Ontario. Sont ensuite proposés des textes qui croisent les regards de différents acteurs sur la diversité ethnoculturelle au sein de l'école et les défis qu'elle soulève, mais aussi les ressources qu'elle constitue pour les relations au sein de l'écologie professionnelle.

\section{Rattachement des auteures}

${ }^{1}$ Université de Montréal, Montréal, Canada; ${ }^{2}$ Université du Québec à Montréal, Montréal, Canada

\section{Correspondance}

joelle.morrissette@umontreal.ca

\section{Mots clés}

enseignants formés à l'étranger, acculturation, stratégies d'insertion, diversité ethnoculturelle, climat scolaire

\section{Pour citer cet article}

Morrissette, J et Audet, G. (2018). L'école à l'épreuve de la diversité ethnoculturelle de son personnel : regards compréhensifs croisés. Alterstice, 8(2), 5-12. 


\section{Introduction et contexte}

Selon l'ONU (Nations unies, s. d.), le nombre de migrations dans le monde (258 millions en 2017) a crû deux fois plus vite au cours de la dernière décennie qu'au cours de la précédente, multipliant ainsi les rencontres interculturelles. Ainsi, les flux migratoires ne cessent d'augmenter, notamment dans les zones urbaines du globe (Organisation de coopération et de développement économiques [OCDE], 2016; Organisation internationale pour la migration [OIM], 2015). S'ensuivent divers changements et mutations de l'ordre social au sein de plusieurs sociétés, qui s'exercent dans une tension entre l'homogénisation et la diversification (Anderson-Levitt, 2003; Maalouf, 2009 ; Mc Andrew, 2010). Cette situation touche particulièrement le Canada, qui est l'État qui reçoit le plus d'immigrants proportionnellement à sa population : en 2017, il a octroyé la résidence permanente à 290000 nouveaux Canadiens (Ministère de l'Immigration, des Réfugiés et de la Citoyenneté du Canada, 2017). L'immigration dans ce pays est toutefois particulière : contrairement à plusieurs autres États, plus vulnérables aux différentes vagues de migrants du fait de leur position géographique, le Canada sélectionne en grande majorité ses immigrants avant leur arrivée au pays. Une large part de ce travail de sélection est dévolue aux provinces, surtout en ce qui concerne le Québec, qui a obtenu du gouvernement fédéral une large autonomie en cette matière. Cette province accueille à elle seule près de $20 \%$ des immigrants au Canada, soit environ 50000 par an (Statistique Canada, 2017), voire davantage avec notamment l'accueil plus récent de réfugiés syriens. La province voisine, l'Ontario, a accueilli 34,7 \% de l'ensemble des immigrants au Canada en 2017 (Ministère des finances de l'Ontario, 2018). Ainsi, le Canada, mène une politique active d'immigration, afin de satisfaire ses besoins en main-d'œuvre et de renouveler sa population décroissante. Dans ce contexte, l'intégration sociale et professionnelle des immigrants et de leurs familles est une question socialement vive, en particulier dans les grands centres urbains, où ils ont davantage tendance à s'établir. Mais c'est à l'école, dans les établissements scolaires et leurs environnements, lieux privilégiés de rencontres, de maillage et de métissage, que cet enjeu prend forme dans des dynamiques de socialisation aux codes du vivre ensemble.

En raison de ce contexte, le champ des rapports interculturels se développe de plus en plus, grâce aux travaux de chercheurs canadiens comme d'ailleurs. Certains portent intérêt à la réussite éducative des élèves issus de l'immigration (Mc Andrew et collab., 2015), aux relations école-famille-communauté en contexte de diversité (Audet, Mc Andrew et Vatz Laaroussi, 2016; Kanouté et Lafortune, 2011; Kanouté et Vatz Laaroussi, 2008; Liboy et Mulatris, 2016), aux représentations de la diversité par les enseignants du groupe majoritaire à leur égard (Bourhis et collab., 2013; Carr et Klassen, 1997) et, en toute logique, à la formation des enseignants à la prise en compte de la diversité ethnoculturelle, linguistique et religieuse (Mc Andrew et Potvin, 2012; SanchezMazas, Changkakoti et Broyon, 2016; Steinbach 2012) ou à leur savoir-faire développé à cet égard (Audet, 2006 et 2011). Dans cette veine, certains étudient le traitement du pluralisme dans les programmes de formation et dans les manuels (Hirsch et Mc Andrew, 2013).

Une attention a été portée plus récemment à la situation des enseignants formés à l'étranger qui obtiennent des contrats ou des postes dans les établissements scolaires de leur société d'accueil, de plus en plus nombreux sur certains territoires, en particulier au Québec où la pénurie actuelle d'enseignants favorise leur embauche. Les travaux qui se penchent sur leurs expériences d'intégration professionnelle s'y intéressent sous l'angle de la transition professionnelle occasionnée par la mobilité géographique (Broyon, 2016; Morrissette et Charara, 2015; Niyubahwe, Makamurera et Jutras, 2014; Oueslati, Mc Andrew et Helly, 2011), en particulier en documentant les difficultés vécues pour s'intégrer à leur nouveau milieu de travail, de même qu'aux stratégies mises en œuvre pour les surmonter (Duchesne, 2017; Elbaz-Luwish, 2004; Myles, Cheng et Wang, 2006; Provencher, Lepage et Gervais, 2016; Remennick, 2002). D’autres travaux étudient la part jouée par la communauté éducative dans leur intégration et leur développement professionnels, adoptant une approche écologique (Gravelle, Duchesne et Gagnon, 2016; Morrissette et Demazière, 2018; Morrissette, Demazière, Diédhiou et Sèguéda 2018; Peeler et Jane, 2005; Phillion, 2003).

De diverses recensions d'écrits ou méta-analyses réalisées jusqu'ici sur ce dernier élément (García et Guerra, 2004; Mc Andrew et collab., 2015; Morrissette, Diédhiou et Charara, 2014; Niyubahwe, Mukamurera et Jutras, 2013; Potvin, Larochelle-Audet, Campbell et Kingué-Longuélé, 2014), il se dégage trois regards dominants. Le premier 
consiste à endosser un angle déficitaire, l'idée de conformité étant sous-jacente : l'immigrant, qu'il soit élève, enseignant, parent ou autre, est "en défaut de savoir " : il se doit d'apprendre les codes et manières de faire communes au sein de la communauté éducative dans laquelle il s'intègre. Le deuxième regard consiste à faire porter tout le poids de l'intégration sur lui, logeant ainsi au second rang - voire occultant - le rôle joué par les différents acteurs de sa nouvelle communauté. Comme le dit Bertheleu (2012), la recherche a adopté jusqu'ici le " paradigme de l'intégration qui tend à focaliser l'attention sur la trajectoire du migrant lui-même [...] en oubliant le contexte de la société d'installation "(p. 31-33). Enfin, le troisième regard est celui de prescriptions et d'orientations à destination des différents acteurs du système éducatif. De fait, et à la faveur des tensions internationales des dernières années, les auteurs proposent différents changements à mettre en place pour que soit mise en œuvre une éducation plus sensible à la diversité, plus inclusive, multi-regards (Loreman, Deppeler et Harvey, 2005; Larochelle-Audet, Borri-Anadon et Potvin, 2016), mais sans pour autant documenter comment ceuxci s'actualisent réellement sur le terrain. Ainsi, de manière générale, on privilégie une posture qui tend à gommer la compétence d'acteur des immigrants, jeunes ou adultes, et qui de ce fait pose un regard réducteur sur l'intégration, ignorant son caractère hautement interactif et laissant dans une boite noire ce qui se passe concrètement au quotidien, sur le terrain, dans les dynamiques socialisantes.

Ce contexte social et scientifique amène à appréhender les dynamiques de métissage qui se nouent au sein de l'école, en particulier dans les centres urbains où la présence de parents, d'élèves, d'enseignants et autres membres du personnel issus de la diversité reconfigure les rapports sociaux. Nous visons à examiner dans ce numéro d'Alterstice les expériences et relations interculturelles au sein de la communauté éducative, ce qui implique de porter attention à un multiplicité d'acteurs : enseignants, élèves, professionnels (orthopédagogues, éducateurs spécialisés, etc.), équipe de direction, employés de soutien (secrétaire, concierge, etc.), familles, organismes communautaires, etc. Sont proposés divers éclairages théoriques et empiriques pour appréhender les processus d'ajustements continus et réciproques dans la négociation de cette diversité à l'école, au travers des interactions quotidiennes. Sont d'abord regroupés des textes qui rapportent spécifiquement la perspective des enseignants formés à l'étranger, pour renseigner leurs expériences d'intégration dans leur nouveau milieu professionnel, au Québec et en Ontario. Ensuite sont rassemblés des textes qui croisent les regards de différents acteurs sur la diversité ethnoculturelle dans l'école, les défis qu'elle soulève ainsi que les ressources qu'elle constitue pour les relations au sein de l'écologie professionnelle.

\section{Expériences d'intégration socioprofessionnelle des enseignants formés à l'étranger}

Les six premiers textes s'intéressent à la diversité au sein de l'équipe-école, principalement celle des pairs enseignants, retenant une vision dynamique qui met en lumière comment cette diversité se négocie dans les interactions avec les autres membres de l'écologie professionnelle.

Adoptant une perspective élargie, Claire Duchesne se penche sur les enjeux et défis en relation avec la langue, la culture et l'identité d'enseignants d'immigration récente dans un contexte francophone minoritaire en Ontario. Reposant sur l'ensemble de ses travaux, la contribution de l'auteure montre que leur intégration à la communauté enseignante ne se fait pas sans difficulté en raison, notamment, de certaines rivalités qui peuvent survenir lorsque les membres du groupe d'enseignants initialement en place ressentent une insécurité linguistique et identitaire devant l'arrivée de nouveaux collègues qu'ils estiment appartenir à un groupe « autre ».

Dans le même ordre d'idées, le texte d'Aline Niyubahwe, Joséphine Mukamurera et Geneviève Sirois traite de la manière dont des enseignants formés à l'étranger vivent les relations interpersonnelles et professionnelles lors de leurs premières années dans des écoles québécoises. II semble que plusieurs défis marquent leurs premières expériences, dont des formes d'isolement, de l'indifférence, voire une certaine méfiance de la part des partenaires de travail. Les auteurs soulignent cependant que les relations nouées en milieu de travail dépendent fortement de certaines caractéristiques contextuelles d'une part, et des qualités personnelles des enseignants formés hors Québec d'autre part. 
La contribution de Joëlle Morrissette, Didier Demazière, Serigne Ben Moustapha Diéhdiou et Saïdou Sèguéda se penche spécifiquement sur les expériences d'enseignants formés à l'étranger s'intégrant dans les écoles montréalaises, à partir de l'étude de ce que ces derniers considèrent comme étant des " chocs " marquant leurs premiers contrats et ce que leurs partenaires de travail voient comme des "faux-pas" de leur part. En ayant convié certains membres de leur écologie professionnelle aux entretiens, les auteurs éclairent ainsi des jeux de socialisation au métier, tel qu'il est conçu et pratiqué au Québec, qui semblent s'actualiser au travers de trois épreuves particulières, qui se situent sur les plans personnel, collectif et institutionnel : celle de l'autonomie, de la modification du rapport de places et de l'enseignement différencié.

À partir de son étude doctorale sur les manières dont le savoir-évaluer des enseignants formés à l'étranger est reconstruit dans leur nouveau milieu travail, Serigne Ben Moustapha Diédhiou expose certains des usages de l'évaluation contestés par les partenaires de travail et qui font obstacle à leur réinvestissement en contexte montréalais. Ainsi, mobiliser l'évaluation pour obliger les élèves à obéir à l'enseignant et valoriser uniquement les élèves les plus performants sont des pratiques rejetées par le milieu d'accueil. L'intérêt de la contribution de l'auteur est d'avoir ainsi révélé des conventions plus ou moins tacites en matière d'évaluation des apprentissages de la culture de travail dans les écoles montréalaises.

L'article de Sony Jabouin et Claire Duchesne identifie aussi des obstacles à l'intégration d'enseignants formés à l'extérieur de l'Ontario, plus précisément des nouveaux enseignants immigrants de minorité visible originaires des Caraïbes et d'Afrique subsaharienne. Utilisant également les résultats de la recherche doctorale de Sony Jabouin, il rend compte spécifiquement des difficultés rencontrées par ces enseignants lors de leur parcours d'insertion professionnelle, en lien avec l'accès à l'emploi, l'affectation et les conditions de la tâche, la socialisation organisationnelle, la professionnalité et l'insertion personnelle et psychologique. Les auteurs mettent ici aussi l'accent sur les stratégies mobilisées par les participants pour surmonter ces difficultés.

Enfin, France Gravelle et Mélissa Bissonnette adoptent un angle différent en présentant, d'une part, le cadre légal qu'une direction d'établissement d'enseignement québécoise doit considérer dans la gestion des relations de travail en contexte de diversité ethnoculturelle et, d'autre part, les résultats de leur recherche documentaire concernant les défis d'insertion socioprofessionnelle du personnel scolaire formé à l'étranger, et donc le rôle précis des directions à cet égard. La finalité de cette contribution consiste en la proposition d'un référentiel de stratégies susceptibles de favoriser l'insertion socioprofessionnelle du personnel scolaire formé à l'étranger.

\section{Regards croisés de différents acteurs sur la diversité ethnoculturelle dans l'école}

Trois contributions composent un second ensemble pour ce numéro.

À partir de sa thèse inscrite dans le paradigme de l'éducation inclusive, Justine Gosselin-Gagné relate ici des observations recueillies dans le quotidien de deux écoles primaires montréalaises situées en contexte de défavorisation socioéconomique et de pluriethnicité. La démarche ethnographique empruntée lui ayant permis d'accéder aux "coulisses » de ces milieux, la chercheuse croise ici le regard des différents acteurs côtoyés pour renseigner d'abord les différents défis propres aux milieux scolaires participants qui ont trait à la précarité, à des difficultés psychosociales, à des traumas et au décodage d'une nouvelle culture scolaire. Puis elle décrit les leviers d'intervention mobilisés par les intervenants des écoles ciblées pour relever ces défis dans l'optique de la réussite éducative, sur quatre plans : le leadership des directions d'école, le climat socioprofessionnel et les pratiques qui visent les élèves, les relations école-familles immigrantes et la collaboration des écoles avec la communauté qui les entoure.

Également intéressées par les populations plus vulnérables, Garine Papazian-Zohrabian, Caterina Mamprin, Vanessa Lemire et Alyssa Turpin-Samson rapportent les résultats d'une recherche-action visant à évaluer une intervention mise en place en contexte scolaire pour favoriser le bien-être et le sentiment d'appartenance des élèves réfugiés syriens. En croisant les perspectives d'élèves et de directions de deux écoles secondaires et une école primaire, les auteurs présentent ici des données secondaires qui mettent en lumière l'importance de la compréhension et de la prise en compte de l'expérience pré, péri et post-migratoire des élèves réfugiés en vue de 
favoriser leur accueil et leur expérience socioscolaire. Ce qui est saisissant de cette étude est l'écart important entre la perception des acteurs scolaires du parcours migratoire et du vécu de leurs élèves réfugiés et de l'expérience réelle de ceux-ci.

Ce numéro s'achève par une contribution collective qui, à l'instar de celle de Justine Gosselin-Gagné, cible le climat d'une école en relation avec la diversité. Ainsi, à partir d'une recension étoffée des écrits, Isabelle Archambault, Marie Mc Andrew, Geneviève Audet, Corina Borri-Anadon, Sivane Hirsch, Valérie Amiraux et Kristel TardifGrenier proposent une définition du climat interculturel. La conceptualisation avancée par les chercheuses comporte cinq dimensions : l'engagement de l'école en faveur d'une culture d'équité et d'ouverture à la diversité dans les rapports avec les élèves, les familles et la communauté, le statut et la légitimité des cultures et des langues d'origine dans les pratiques en classe et dans les normes des établissements, les attitudes du personnel à l'égard des élèves et des familles d'origines diverses, la qualité des relations interculturelles entre les élèves et le personnel d'origines diverses, et le soutien de l'école à la construction identitaire des jeunes issus de la diversité.

\section{Conclusion}

Comme les lecteurs l'auront bien vu, plusieurs de nos contributeurs sont de jeunes chercheurs, ce qui suggère fortement que la question de la diversité ethnoculturelle à l'école est une thématique de pointe, en cohérence avec les défis de l'évolution de nos sociétés. Nous avons souhaité ici croiser les points de vue, mais il ne s'agit que d'un premier pas : les prospectives en recherche nous semblent loger à l'enseigne de cadres théoriques novateurs, aux croisements de disciplines et d'approches méthodologiques plus ambitieuses, susceptibles de mieux appréhender la complexité actuelle des phénomènes interculturels.

\section{Références bibliographiques}

Anderson-Levitt, K. (2003). Local Meanings, Global Schooling. Anthropology and World Culture Theory. New York: Palgrave Macmillan.

Audet, G. (2006). Pour une " altérité en acte » : reconstruction et théorisation de récits de pratique d'éducation interculturelle en maternelle (Thèse de doctorat inédite). Québec, Université Laval.

Audet, G. (2011). Composer avec la diversité culturelle en classe de maternelle : résultats d'une analyse de récits de pratique enseignante. Revue de l'intégration et des migrations internationales, 12(1), 43-60.

Audet, G., Mc Andrew, M. et Vatz Laaroussi, M. (2016). Partnership between schools and immigrant families and communities. Dans C. Timmerman, N. Clycq, J. Haers, M. Mc Andrew, S. Mels et A. Balde (dir.), Youth, education and value change: The school as a context of educational success? (p. 215-232). London: Routledge.

Bertheleu, H. (2012). Discriminations et relations interethniques : quelques remarques. Dans R. Guyon (dir.), Des différences (im)-pertinentes. Diversité. Ville école intégration diversité enjeux, 168, 31-36.

Bourhis, R. et collab. (2013). Sécurité identitaire et attitudes à l'égard de l'Autre chez de futurs enseignants: les effets d'une formation interculturelle. Dans M. Mc Andrew, M. Potvin et C. Anadon (dir.), Le développement d'institutions inclusives en contexte de diversité (p. 117-132). Québec : Presses de l'Université du Québec.

Broyon, M.A. (2016). L'insertion professionnelle des enseignants issus de la migration en Suisse romande : une insertion comme les autres ? Formation et pratiques d'enseignement en questions, 21, 39-58. http://revuedeshep.ch/pdf/21/21-03-Broyon.pdf

Carr, P.R. et Klassen, T.R. (1997). Different perceptions of race in education: Racial minority and white teachers. Canadian Journal of Education, 22(1), 67-81.

Duchesne, C. (2017). Quelles stratégies d'acculturation de nouveaux enseignants issus de l'immigration privilégientils face aux défis culturels et identitaires de leur insertion professionnelle ? Revue canadienne de l'éducation, 40(1), 1-24. http://journals.sfu.ca/cje/index.php/cje-rce/article/view/2327/2381 
Elbaz-Luwisch, F. (2004). Immigrant teachers: stories of self and place. International Journal of Qualitative Studies in Education, 17(3), 387-414.

https://www.tandfonline.com/doi/pdf/10.1080/0951839042000204634?needAccess=true

García, S. B. et Guerra, P. L. (2004). Deconstructing deficit thinking : Working with educators to create more equitable learning environments. Education and Urban Society, 36(2), 150-168. http://citeseerx.ist.psu.edu/viewdoc/download?doi=10.1.1.923.2705etrep=rep1ettype=pdf

Gravelle, F., Duchesne, C. et Gagnon, N. (2016). Stratégies d'encadrement efficientes pour l'insertion professionnelle des nouveaux enseignants issus de l'immigration. Actes du colloque international du RIFEFF, "Mieux former les enseignants dans la francophonie: principaux enjeux actuels et futures $॥$, 563-573.

Hirsch, S. et Mc Andrew, M. (2013) La représentation de la communauté juive dans les manuels scolaires québécois. Nouveaux Cahiers de Recherche en Éducation, 15(2), 34-63. https://www.erudit.org/fr/revues/ncre/2012-v15-n2-ncre0824/1018456ar/

Kanouté, F. et Lafortune, G. (2011). La réussite scolaire des élèves d’origine immigrée: réflexions sur quelques enjeux à Montréal. Éducation et Francophonie, 39(1), 80-92. https://www.erudit.org/fr/revues/ef/2011-v39n1-ef1807263/1004331ar/

Kanouté, F. et Vatz Laaroussi, M. (2008). La relation écoles-familles immigrantes : une préoccupation récurrente, et pertinente. Revue des sciences de l'éducation, 34(2), 259-264. https://www.erudit.org/fr/revues/rse/2008v34-n2-rse2553/019680ar/

Larochelle-Audet, J. Borri-Anadon, C. et Potvin, M. (2016). La formation interculturelle et inclusive du personnel enseignant : conceptualisation et opérationnalisation de compétences professionnelles. Éducation et francophonie, 44(2), 172-195. https://www.erudit.org/en/journals/ef/2016-v44-n2ef02953/1039027ar/abstract/

Liboy, G.-M. et Mulatris, P. (2016). Enseignants non immigrants et enseignants immigrants : convergences et divergences autour de la relation entre école et familles immigrantes. Alterstice, 6(1), 91-104. https://www.erudit.org/fr/revues/alterstice/2016-v6-n1-alterstice02867/1038282ar.pdf

Loreman, T., Deppeler, J. et Harvey, D. (2005). Inclusive Education: A Practical Guide to Supporting Diversity in the Classroom. United Kingdom : Routledge Falmer.

Maalouf, A. (2009). Le dérèglement du monde. Paris : Grasset.

Mc Andrew, M. (2010). Les majorités fragiles et l'éducation. Montréal : Presses de l'Université de Montréal.

Mc Andrew, M., Balde, A., Bakhshaei, M., Tardif-Grenier, K., Audet, G., Armand, F., Guyon, S., Ledent, J., Lemieux, G., Potvin, M., Rahm, J., Vatz Laaroussi, M., Carpentier, A. et Rousseau, C. (2015). La réussite éducative des élèves issus de l'immigration. Dix ans de recherche et d'intervention au Québec. Montréal : Presses de l'Université de Montréal.

Mc Andrew, M. et Potvin, M. (2012). Portrait de l'offre de formation initiale sur la diversité ethnoculturelle, religieuse et linguistique dans les programmes destinés au personnel scolaire des universités québécoises. Montréal : DSCC/MELS-CEETUM-CHEREUM.

Ministère des finances de l'Ontario (2018). Rapport démographique trimestriel de l'Ontario : Faits saillants du premier trimestre 2018. https://www.fin.gov.on.ca/fr/economy/demographics/quarterly/dhiq1.html

Ministère de l'Immigration, des Réfugiés et de la Citoyenneté. (2017). Rapport annuel au parlement sur l'immigration. Ottawa : Immigration, Réfugiés et Citoyenneté Canada. https://www.canada.ca/fr/immigration-refugies-citoyennete/organisation/publications-guides/rapportannuel-parlement-immigration-2017.html

Morrissette, J., Charara, Y. (2015). Le groupe de codéveloppement professionnel: un levier de réflexivité pour l'intégration socioprofessionnelle des enseignants formés à l'étranger. Questions vives, 24, 19 p. https://questionsvives.revues.org/1805 
Morrissette, J. et Demazière, J. (2018). Dualité des processus de socialisation professionnelle des enseignants formés hors Québec. Entre imposition et appropriation. Alterstice, 8(1), 95-106.

https://www.journal.psy.ulaval.ca/ojs/index.php/ARIRI/article/view/orrissette_Alterstice8\%281\%29

Morrissette, J., Demazière, D., Diédhiou, S.B.M. et Sèguéda, S. (2018). Les expériences d'intégration professionnelle d'enseignants migrants à Montréal. Rapport de recherche soutenu par le Conseil de recherche en sciences humaines du Canada et la Commission scolaire Marguerite-Bourgeois, Montréal. https://cipcd.ca/wpcontent/uploads/2014/04/Rapport-de-recherche-Morrissette-Demazi\%C3\%A8re-intergrationprofessionnelle-ens-migrants.pdf

Morrissette, J., Diédhiou, B. et Charara, Y. (2014). Un portrait de la recherche sur l'intégration socioprofessionnelle des enseignants formés à l'étranger. Rapport de recherche déposé au Centre d'intervention pédagogique en contexte de diversité de la Commission scolaire Marguerite-Bourgeois, Montréal. http://www.cipcd.ca/wpcontent/uploads/2014/10/Rapport-Morrissette-et-al-VF-20-oct-2014.pdf

Myles, J., Cheng, L. et Wang, H. (2006). Teaching in elementary school: Perceptions of foreign- trained teacher candidates on their teaching practicum. Teaching and Teacher Education, 22(2), 233-245.

Nations unies (s. d.). Migrations. https://www.un.org/fr/sections/issues-depth/migration/

Niyubahwe A., Makamurera J. et Jutras F. (2013). Professional integration of immigrant teachers in the school system: A literature review. McGill Journal of Education, 48(2), 279-296.

http://mje.mcgill.ca/article/viewFile/8879/6876

Niyubahwe A., Makamurera J. et Jutras F. (2014). L'expérience de transition professionnelle des enseignants de migration récente au Québec. Revue canadienne de l'éducation, 37(4), 1-32.

http://journals.sfu.ca/cje/index.php/cje-rce/article/view/1724/1719

Organisation internationale pour la migration (OIM). (2015). État de la migration dans le monde 2015. Les migrants et les villes : de nouveaux partenariats pour gérer la mobilité. www.iom.int/fr/etat-de-la-migration-dans-lemonde-2015

Organisation de coopération et de développement économiques (OCDE). (2016). Perspectives des migrations internationales 2016. Paris : OCDE.

Oueslati, B., Mc Andrew, M. et Helly, D. (2011). Islam and Muslim cultures in Quebec French-language textbooks over three periods: 1980s, 1990s, and the present day. Journal of Educational Media, Memory, and Society, 3(1), 5-24.

Peeler, E. et Jane, B. (2005). Mentoring: Immigrant teachers bridging professional practices. Teaching Education, 16(4), 325-336.

Phillion, J. (2003). Obstacles to accessing the teaching profession for immigrant women. Multicultural Education, 1(11), 41-45.

Potvin, M., Larochelle-Audet, J., Campbell, M.-E. t Kingué-Longuélé, G. (2014). L'éducation interculturelle inclusive, antiraciste et à la citoyenneté. Recension analytique de la littérature sur la prise en compte de la diversité ethnoculturelle, religieuse et linguistique en éducation. Rapport final soumis à la Direction des Services aux communautés culturelles, Montréal.

Provencher, A., Lepage, M. et Gervais, C. (2016). Difficultés éprouvées dans la maitrise de certaines compétences professionnelles chez des enseignantes-stagiaires issues de l'immigration récente. Formation et Profession, 24(1), 16-28.

Remennick, L. (2002). Survival of the fittest: Russian immigrant teachers speak about their professional adjustment in Israel. International Migration, 40(1), 99-121.

Sanchez-Mazas, M., Changkakoti, N. et Broyon, M.-A. (dir.). (2016). Éducation à la diversité. Décalages, impensés, avancées. Paris : L'Harmattan. 
Statistique Canada. (2017). 2017 : I'année en statistiques. Ottawa : Gouvernement du Canada. https://www.statcan.gc.ca/fra/blogue/sc/2017-annee-stats

Steinbach, M. (2012). Élargir les perspectives interculturelles des futurs enseignants. Mc Gill Journal of Education, 47(1), 153-170. http://mje.mcgill.ca/index.php/MJE/article/viewFile/8824/6812 